\title{
Pyramidal ice crystal scattering phase functions and concentric halos
}

\author{
C. Liu, P. R. Jonas, C. P. R. Saunders \\ Department of Pure and Applied Physics, UMIST, PO Box 88, Manchester M60 1QD, UK
}

Received: 8 June 1995/Revised: 23 May 1996/Accepted: 6 June 1996

\begin{abstract}
Phase functions have been calculated using the Monte Carlo/geometric ray tracing method for single hexagonal pyramidal ice crystals (such as solid and hollow bullets) randomly oriented in space and horizontal plane, in order to study the concentric halo formations. Results from three dimensional model calculations show that $9^{\circ}$ halo can be as bright as the common $22^{\circ}$ halo for pyramidal angle of $28^{\circ}$, and the $18^{\circ}, 20^{\circ}, 24^{\circ}$ and $35^{\circ}$ halos cannot be seen due to the strong $22^{\circ}$ halo domination in the scattering phase function between $18^{\circ}$ and $35^{\circ}$. For solid pyramidal ice crystals randomly oriented horizontally, the $35^{\circ}$ arc can be produced and its intensity depends on the incident ray solar angle and the particle aspect ratio.
\end{abstract}

\section{Introduction}

Most of the atmospheric optical phenomena are caused by ice crystals shaped like plates or columns, with hexagonal cross sections. Refraction of the Sun's rays by such crystals when randomly oriented in space accounts for the common $22^{\circ}$ halo, and also the much less common $46^{\circ}$ halo. However, the ordinary hexagonal crystal cannot explain the origin of halos of unusual radii as reported by Goldie et al. (1976) and Neiman (1989) who observed simultaneously as many as six concentric halos including $9^{\circ}, 18^{\circ}, 20^{\circ}, 24^{\circ}, 35^{\circ}, 22^{\circ}$ and $46^{\circ}$ halos. Goldie et al. (1976) and Neimann (1989) believed that the $9^{\circ}, 18^{\circ}, 20^{\circ}, 24^{\circ}$ and $35^{\circ}$ halos are basically from the solid pyramidal ice crystals (columns with two pyramidal tops) in the upper troposphere. Neiman also explained the origination of the observed halos by calculating the halo angle values using the simple minimum deviation formula (Minnaert, 1954; Tricker, 1970), and the calculated results are in good agreement with the observed values.

Correspondence to: C. Liu
The concentric halos with unusual radii have been reported many times since the beginning of this century, such as by Andrus (1915), Kimball (1915), Brush (1919), Leaf (1926) and Rechardson (1953), but it is very rare to see them. Sassen et al. (1994) have tried to explain why the common halo is not more common in cirrus clouds with the aid of new ray-tracing simulations for hexagonal hollow-ended column and bullet-rosette models, and concluded that the typical cirrus particles from in situ observations are too hollow or complex to produce vivid halos. Of course, there are many other types of ice crystal shapes existing in cirrus cloud as observed by Mossop and Ono (1968), Heymsfield (1975) and Heymsfield and Platt (1984) in field measurements and by Magono and Lee (1966) from laboratory observations.

In the last decade, the ray-tracing method has been developed and employed by Wendling et al. (1979), Coleman and Liou (1981), Cai and Liou (1982), Takano and Jayaweera (1985), Rockwitz (1989), Muinonen et al. (1989), and Takano and Liou (1989) to calculate the ice crystal scattering phase functions for different ice crystal shapes, such as hexagonal column- and plate-like ice crystal. This method has been extended to study the scattering phase functions for more complicated ice crystal shapes (Macke and Tzschichholz, 1993; Macke, 1993; Liou and Takano, 1994; Iaquinta et al., 1995; Liu et al., 1995). Some pyramidal particles have also been studied by Takano and Liou (1995) using a new Monte Carlo/ray-tracing method. Based on the ray-tracing results, Takano and Liou (1989) have discussed numerous optical features observed in the presence of cirrus, including the halos produced from the three-dimensional ice crystal orientation and the arcs from two-dimensional ice crystal orientation. All this progress has made it possible to calculate the pyramidal ice crystal scattering phase function quantitatively, and to study the halo formation systematically.

In this study we try to investigate the concentric halo formation systematically by extending Neiman's (1989) work to other pyramidal particles using minimum deviation formula, and quantitatively by modelling the hexagonal pyramidal ice particle phase functions using 
a Monte Carlo/ray-tracing method. In our model, the particle is assumed to be randomly oriented in space and in horizontal plane, respectively. The pyramidal angle $\delta$ (defined as the angle between the side face of the pyramid and its vertical axis) is assumed to be $28^{\circ}$, the incident light wavelength is $0.55 \mu \mathrm{m}$, and the ice particle refractive index at this wavelength is $1.3106-i 3.11 \times 10^{-9}$ (Warren, 1984).

\section{Halo positions}

The particle geometrical structures considered in this paper are shown in Fig. 1, they are (a) solid column, (b) hollow column (column with two pyramidal cavities), (c) solid pyramidal particle (column with two pyramidal tops), (d) solid bullet (column with one pyramidal top), and (e) hollow bullet (column with one pyramidal top and one pyramidal cavity). $\mathrm{A}$ and $\mathrm{C}$ are column radius and length, respectively, and $\mathrm{H}$ is the pyramidal top or cavity height. The horizontal hexagonal cross section and the face number order of these particles are shown in Fig. 1f. The upper part (including top or cavity) of the pyramidal particle can be defined as part $\mathrm{L}$, the middle column part as part $\mathrm{M}$, and the lower part as part $\mathrm{N}$ as shown in Fig. 1c.

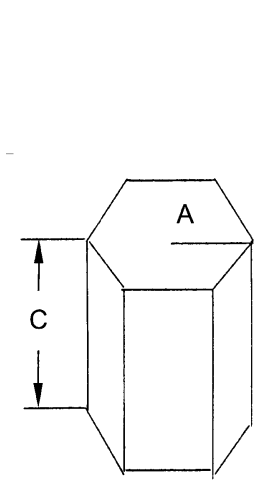

a
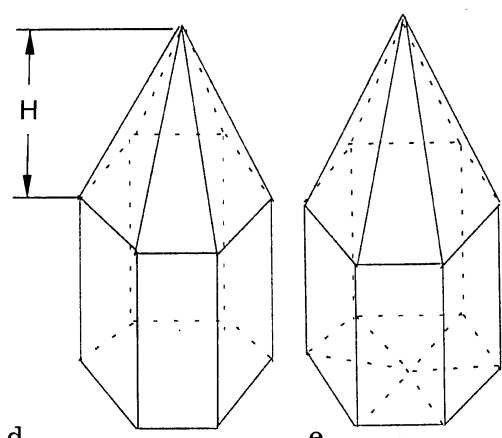

e

b
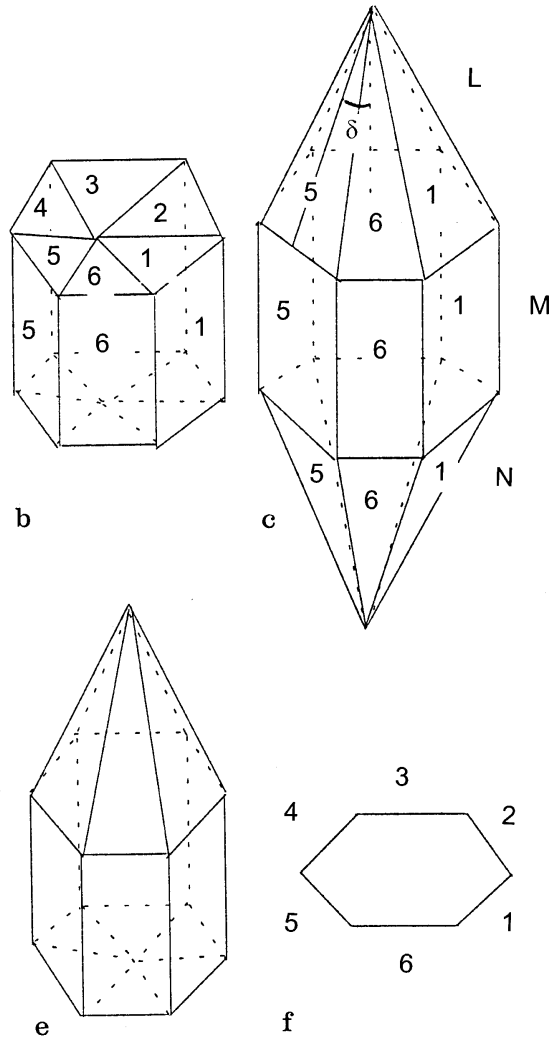

Fig. 1a-f. Diagram showing pyramidal particle geometrical structures. a Solid column; b hollow column; c solid pyramidal particle; d solid bullet; e hollow bullet, and f face number order (see text)
Table 1. Halo positions

\begin{tabular}{llll}
\hline Particle type & $\begin{array}{l}\text { Ray entrance } \\
\text { and exit faces }\end{array}$ & $\begin{array}{l}\text { Prism angle } \\
\text { (degrees) }\end{array}$ & $\begin{array}{l}\text { Halo position } \\
\text { (degrees) }\end{array}$ \\
\hline b & L1M4 & 28 & 9 \\
b & L1N3 & 52.4 & 18 \\
b & L1M3 & 63.8 & 24 \\
c & L1N3 & 52.4 & 18 \\
d & L1L3 & 80.25 & 35 \\
d & L1L4 & 56.0 & 20 \\
d & L1M3 & 63.8 & 24 \\
d & L1M4 & 28 & 9 \\
d & L1N & 62 & 23 \\
e & L1N2 & 52.4 & 18 \\
\hline
\end{tabular}

Goldie et al. (1976) and Greenler (1980) pointed out that the size of halos with unusual radii is governed by the pyramidal angle $\delta$ and the prism angle $\beta$ (angle between two pyramidal faces). Goldie et al. (1976) concluded from previous ice crystal microphysical studies and from the photographs they collected while documenting the 1974 concentric halo display that $\delta=28^{\circ}$, which will be used in following model calculations and in the minimum deviation formula

$D_{\min }=2 \sin ^{-1}\left(m_{r} \sin \frac{\beta}{2}\right)-\beta$

where $D_{\min }$ is the minimum deviation angle. This equation holds only for incident angle (defined as an acute angle between the prism face normal and the incident ray direction) so chosen that the light ray passes symmetrically through the prism, and it is the minimum value with respect to rotation of the prism. The real part of the refractive index $\left(m_{r}\right)$ used here is 1.3106 , and the maximum refraction angle for this value is $49.73^{\circ}$, so the minimum deviation does not exist when $\beta>99.5^{\circ}$ for the wavelength under consideration.

Similar to Neiman (1989), the possible halo positions are listed in Table 1 . For column, the strong $22^{\circ}$ and weak $46^{\circ}$ halos are well known, so they are not listed again. Due to symmetrical structure of the hexagonal ice particle, only limited halos appear, and some of the duplicated ray processes are not listed in Table 1. Except particle type (d) gives extra $23^{\circ}$ halo which does not appear in Neiman's table, other halo positions have already been predicted by Neiman. So, the possible halo positions are $9^{\circ}, 18^{\circ}, 20^{\circ}$, $23^{\circ}, 24^{\circ}$, and $35^{\circ}$.

\section{Model results}

In order to study the halo intensity quantitatively, phase functions of these pyramidal particles are calculated, by adding reflection, refraction and diffraction together, and all phase functions are normalized to 1.0. In order to see the phase function shape clearly, the maximum of the $\mathrm{y}$ axis is set to be 10 , so the value at zero scattering angle will be cut off. In following model calculations, the column 
radius is $\mathrm{A}=30 \mu \mathrm{m}$ and length $\mathrm{C}=300 \mu \mathrm{m}$ for all particles if not specified, and the pyramidal angle $\delta=28^{\circ}$ for both pyramidal top and cavity. The photon number is $10^{7}$ for each phase function calculation, and the scattering angle resolution is $1^{\circ}$.

\section{$3.13 D$ model results}

The calculated phase functions for particles randomly oriented in space are shown in Fig. 2. The broken line is the phase function of solid column, and the solid lines are phase functions for (a) solid bullet, (b) hollow bullet, (c) hollow column, and (d) solid pyramidal particle. It can be seen that besides the strong $22^{\circ}$ halo resulting from the particle hexagonal structure, there is another $9^{\circ}$ halo resulting from the particle pyramidal top or cavity structures, and for both hollow bullet and hollow column, the $9^{\circ}$ halo intensity is comparable with that of $22^{\circ}$ halo. It is also noted that the hollow bullet, hollow column and solid pyramidal particles have lower backscattering than that of solid column.

For pyramidal particles randomly oriented in space, the $9^{\circ}, 22^{\circ}$, and $46^{\circ}$ halos can be seen, but there are no $18^{\circ}$, $20^{\circ}, 23^{\circ}, 24^{\circ}$, and $35^{\circ}$ halo structures, this may be due to the strong $22^{\circ}$ halo domination in the scattering phase function between $18^{\circ}$ and $35^{\circ}$.

\section{$3.22 \mathrm{D}$ model results}

According to observational results (Ono, 1969), the ice crystal particles are generally oriented in the cloud with their major axes parallel to the ground. Lidar measurements for ice clouds further illustrated the preferred orientation of the plate-type crystals (Platt, 1978). In order to investigate the particle orientation effect on some optical features, the horizontally oriented ice particle scattering phase function will be calculated. The horizontally oriented particle is shown in Fig. 3, and it is assumed that the particle can rotate randomly around $\mathrm{x}$ axis (along particle's long axis parallel to the ground) or $\mathrm{z}$ axis (assumed to be vertical), but it does not rotate along y axis (horizontal). These rotation limitations will make particle asymmetrically oriented relative to the incident ray, so the 2D model can only produce arcs, rather than concentric halos as produced in 3D model. The angle between the incident ray and the vertical axis is $\theta$. The calculated phase functions for particles randomly oriented in the horizontal plane are shown in Fig. 4.

The broken line in Fig. 4 is the phase function for solid column randomly oriented in space, and the solid lines are phase functions for solid pyramidal ice particle (Fig. 1c)

Fig. 2a-d. Phase function angular distribution for particles randomly oriented in space. The broken line is for solid column with the column radius $\mathrm{A}=30 \mu \mathrm{m}$, and length $\mathrm{C}=300 \mu \mathrm{m}$. The solid line is for a solid bullet; b hollow bullet; c hollow column, and d solid pyramidal particle
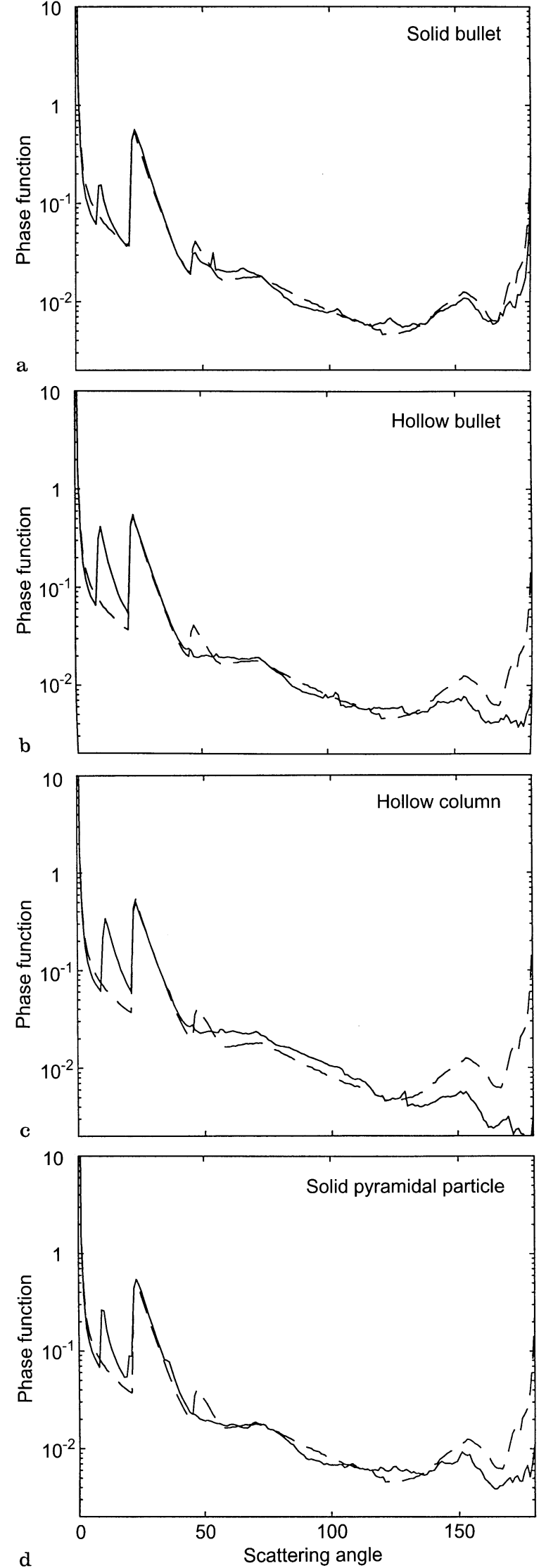
randomly oriented in the horizontal plane. Four cases are considered here: (a) $\theta=10^{\circ}$, (b) $\theta=30^{\circ}$, (c) $\theta=60^{\circ}$, and (d) $\theta=30^{\circ}$, but for pyramidal particle column radius $\mathrm{A}=30 \mu \mathrm{m}$ and column length $\mathrm{C}=100 \mu \mathrm{m}$. The $35^{\circ}$ arc can be seen, and its intensity is as weak as that of the $46^{\circ}$ halo. It is also noted that the $35^{\circ}$ arc intensity varies with the incident angle $\theta$, and this is also true for $9^{\circ}$ and $22^{\circ}$ halos. This is because a smaller percentage of rays will undergo the processes confined by Eq. (1) to produce these peaks as $\theta$ increases, and the energy will be directed to other scattering angles, which can be seen clearly from the phase function shape variations throughout the whole scattering angle range.

After comparing Fig. $4 \mathrm{~b}$ and d, it can be seen that for the same incident angle of $\theta=30^{\circ}$, the smaller the particle aspect ratio $(\mathrm{C} / \mathrm{A})(\mathrm{Fig} .4 \mathrm{~d})$, the stronger the $35^{\circ}$ arc, this is because of the decrease of the pyramidal column length, there will be a smaller percentage of rays undergoing processes producing $22^{\circ}$ halo and forward zero angle peak, so the $22^{\circ}$ halo and zero angle intensity will be decreased and the scattering intensity at other scattering angles will be increased relatively due to the normalization. Furthermore, the $18^{\circ}, 20^{\circ}, 23^{\circ}$, and $24^{\circ}$ arcs can also be seen, but they are very difficult to distinguish from $22^{\circ}$ halo.

Phase functions for other particles randomly oriented in the horizontal plane are also calculated and plotted (solid lines) in Fig. 5 for: (a) solid bullet, (b) hollow bullet, (c) hollow column, and (d) solid pyramidal particle, together with the phase function of solid column randomly oriented in space (broken line). The incident ray angle is $\theta=30^{\circ}$. It can be seen that only the solid pyramidal particle can produce recognizable $35^{\circ}$ arc, but it is very weak.

\section{Conclusions}

Several pyramidal ice crystal particle shapes have been considered to study the formation of concentric halos and some arcs, using Monte Carlo/ray-tracing method. The

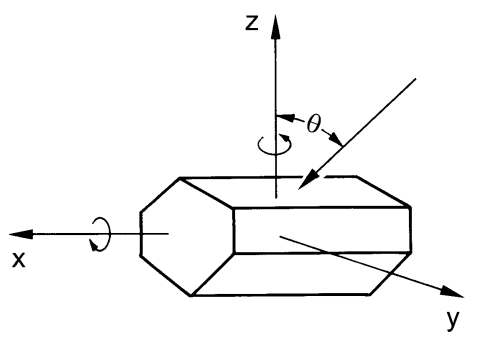

Fig. 3. Diagram showing particle randomly oriented in horizontal plane

Fig. 4a-d. Phase function angular distribution. The broken line is for solid column randomly oriented in space, with the column radius $\mathrm{A}=30 \mu \mathrm{m}$, and length $\mathrm{C}=300 \mu \mathrm{m}$. The solid line is for solid pyramidal particle randomly oriented in horizontal plane, with the incident angle: a $\theta=10^{\circ}$; b $\theta=30^{\circ}$; c $\theta=60^{\circ}$, and d $\theta=30^{\circ}$, but for column length $\mathrm{C}=100 \mu \mathrm{m}$
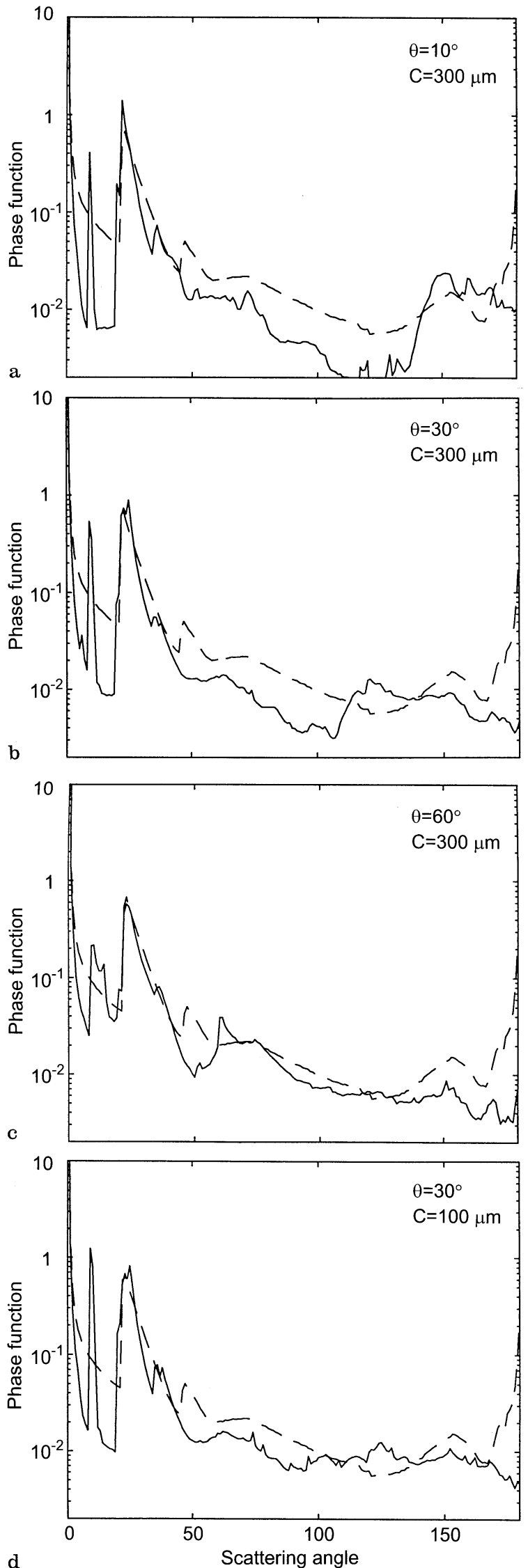

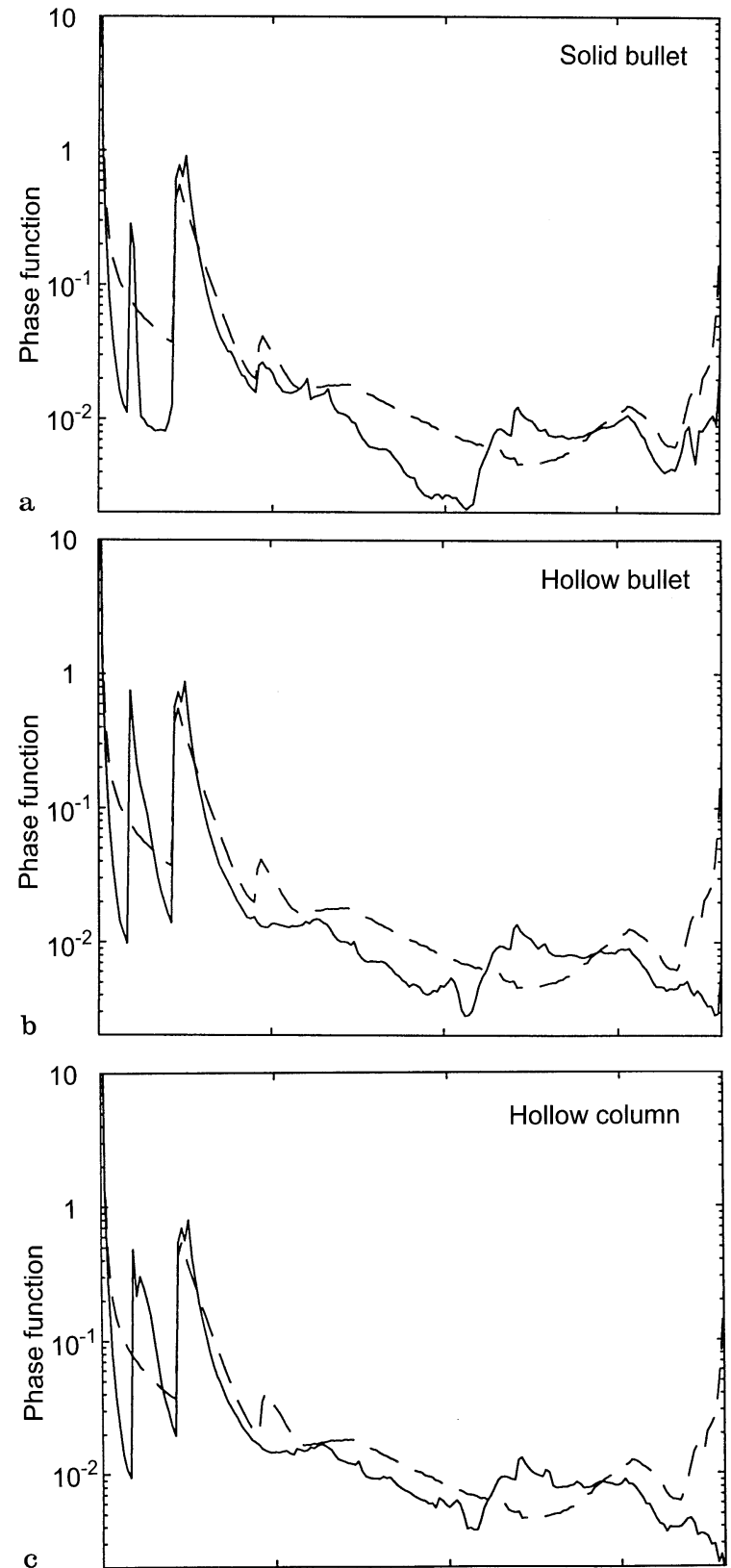

c

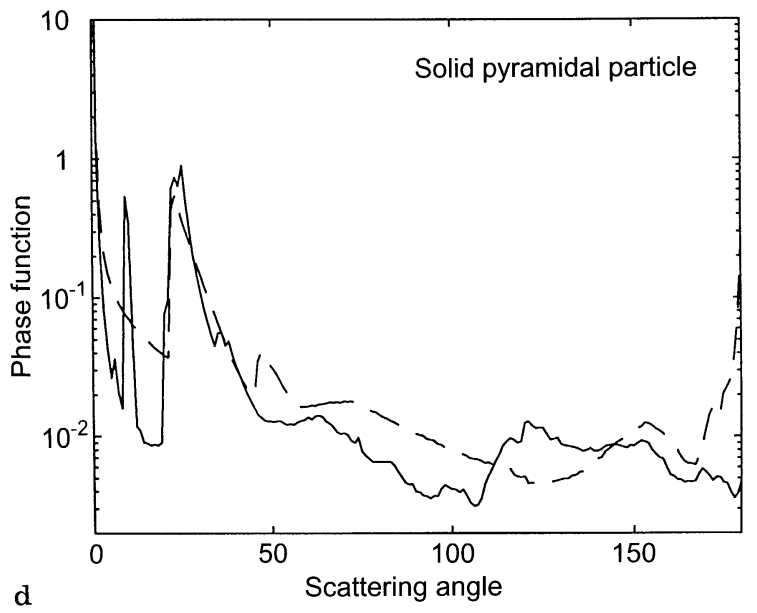

halo positions were investigated using minimum deviation formula, and the halo and arc intensities were calculated quantitatively by modelling the particle scattering phase functions for particles randomly oriented in space and in the horizontal plane, respectively.

Throughout our model studies, it can be seen that the solid pyramidal particle randomly oriented in space can only produce $9^{\circ}, 22^{\circ}$, and $46^{\circ}$ halos theoretically, other halos cannot be seen due to $22^{\circ}$ halo dominations between $18^{\circ}$ and $35^{\circ}$. When the solid pyramidal particle is randomly oriented in the horizontal plane, some arcs, such as $18^{\circ}$, $20^{\circ}, 23^{\circ}, 24^{\circ}$, and $35^{\circ}$ arcs, can be produced. The rare occurrence of these halos in laboratory and in nature needs further study (Sassen et al., 1994). The particle formation, its external and internal structures and its orientation, have to be further investigated before giving any useful conclusion.

Acknowledgements. The work described in this paper has been undertaken with support from the NERC under Research Grant GST/02/586. We are grateful to two referees for their valuable comments.

Topical Editor L. Eymard thanks K. Sassen and A. Macke for their help in evaluating this paper.

\section{References}

Andrus, C. G., Solar halo of 11 May 1915, at Sand Key, Florida, Mon. Weather Rev., 43, 213-214, 1915.

Brush, J. W., Quintuple solar halos, Mon. Weather Rev., 47, 340, 1919.

Cai, Q., and K. N. Liou, Polarized light scattering by hexagonal ice crystals: theory, Appl. Opt., 21, 3569-3580, 1982.

Coleman, R. F., and K. N. Liou, Light scattering by hexagonal columns and plates, J. Atmos. Sci., 38, 1260-1271, 1981.

Goldie, E. C. W., G. T. Meaden, and R. White, The concentric halo display of 14 April 1974, Weather, 31, 304-311, 1976.

Greenler, R., Rainbows, halos, and glories, Cambridge University Press, New York, 1980.

Heymsfield, A. J., Cirrus uncinus generating cells and the evolution of cirriform clouds. Part 1. Aircraft observations of the growth of the ice phase, J. Atmos. Sci., 23, 799-808, 1975.

Heymsfield, A. J., and C. M. R. Platt, A parametrization of the particle size spectrum of ice clouds in terms of the ambient temperature and the ice water content, J. Atmos. Sci., 41, 846-855, 1984.

Iaquinta, J., H. Isaka, and P. Personne, Scattering phase function of bullet rosette ice crystals, J. Atmos. Sci., 52, 1401-1413, 1995.

Kimball, J. H., Lunar halo of 24-25 June 1915, at Richmond, VA, Mon. Weather Rev., 43, 592, 1915.

Leaf, C., Rare halos, Meteorol. Mag. 61, 111, 1926.

Liou, K. N., and Y. Takano, Light scattering by nonspherical particles: remote sensing and climatic applications, Atmos. Res., 31, 271-298, 1994.

Liu, C., P. R. Jonas, and C. P. R. Saunders, Phase function calculations of single hexagonal bullet-like ice crystals, Ann. Geophysicae, 13, 1348-1354, 1995.

Fig. 5a-d. Phase function angular distribution. The broken line is for solid column randomly oriented in space, with the column radius $\mathrm{A}=30 \mu \mathrm{m}$, and length $\mathrm{C}=300 \mu \mathrm{m}$. The solid line is for particles randomly oriented in horizontal plane: a solid bullet; b hollow bullet; $\mathbf{c}$ hollow column, and $\mathbf{d}$ solid pyramid particle. The incident ray angle is $\theta=30^{\circ}$ 
Macke, A., Scattering of light by polyhedral ice crystals, Appl. Opt., 32, 2780-2788, 1993.

Macke, A., and F. Tzschichholz, Scattering of light by fractal ice particles: a qualitative estimate exemplary for two-dimensional triadic Koch-islands, Physica A, 191, 545-548, 1993.

Magono, C., and C. W. Lee, Meteorological classification of natural snow crystals, J. Faculty of Science, Hokkaido University, Japan. Series VII (Geophysics), 2, (4), 1996.

Minnaert, M., The nature of light and colour in the open air, Dover, New York, 1954.

Mossop, S. C., and A. Ono, Measurements of ice crystal concentration in clouds, J. Atmos. Sci., 26, 130-137, 1968.

Muinonen, K., K. Lumme, J. Peltoniemi, and M. I. William, Light scattering by randomly oriented crystals, Appl. Opt., 28, 3051-3060, 1989 .

Neiman, J. N., The Boulder, Colorado, concentric halo display of 21 July 1986, Bull. Am. Meteorol. Soc., 70, 258-264, 1989.

Ono, A., The shape and riming properties of ice crystals in natural clouds, J. Atmos. Sci., 26, 138-147, 1969.

Platt, C. M. R., Lidar backscattering from hexagonal ice crystal plates, J. Appl. Meteorol., 17, 482-488, 1978.

Rechardson, W. E., Four simultaneous concentric halos, Meteorol. Mag. 82, 277-279, 1953.
Rockwitz, K. D., Scattering properties of horizontally oriented ice crystal columns in cirrus clouds, Part I, Appl. Opt., 28, 4103-4110, 1989.

Sassen, K., N. C. Knight, Y. Takano, and A. J. Heymsfield, Effects of ice-crystal structure on halo formation: cirrus cloud experimental and ray-tracing modelling studies, Appl. Opt., 33, 4590-4601, 1994.

Takano, Y., and K. Jayaweera, Scattering phase matrix for hexagonal ice crystals computed from ray optics, Appl. Opt., 24, 3254-3263, 1985.

Takano, Y., and K. N. Liou, Solar radiation transfer in cirrus clouds. Part I: single-scattering and optical properties of hexagonal ice crystals, J. Atmos. Sci., 46, 3-19, 1989.

Takano, Y., and K. N. Liou, Radiation transfer in cirrus clouds. Part III: light scattering by irregular ice crystals, J. Atmos. Sci., 52, 818-837, 1995.

Tricker, R. A. R., Introduction to meteorological optics, Elsevier, New York, 1970.

Warren, S. G., Optical constants of ice from the ultraviolet to the microwave, Appl. Optics., 23, 1206-1225, 1984.

Wendling, P., R. Wendling, and K. K. Weickmann, Scattering of solar radiation by hexagonal ice crystals, Appl. Optics, 18, 2663-2671, 1979. 